\title{
Corticosteroids in diabetes patients infected with COVID-19
}

\section{Fuxue Deng ${ }^{1} \cdot$ Dengfeng Gao ${ }^{1} \cdot$ Xiaorong $\mathrm{Ma}^{2} \cdot$ Yonghong Guo ${ }^{3} \cdot$ Rong Wang $^{1} \cdot$ Wei Jiang ${ }^{1}$ (D) Shouping Gong ${ }^{4}$}

Received: 14 June 2020 / Accepted: 18 June 2020 / Published online: 25 June 2020

(C) Royal Academy of Medicine in Ireland 2020

Coronavirus disease 2019 (COVID-19) has now become a worldwide pandemic, and infected countries and districts face extraordinary challenges in constraining the rapid spread of the virus. Nearly a million people have been infected as of April 1, and 42,000 have died worldwide since the December 2019 outbreak in Wuhan, China. The number is still increasing daily at an exponential growth rate. According to the published paper and statistics from the Centers for Disease Control and Prevention (CDC) and the World Health Organization (WHO), severe cases account for nearly $10 \%$, and $5 \%$ of patients need mechanical ventilation. Death often occurs in elder people with comorbidities, such as hypertension, coronary heart disease, or diabetes [1].

\section{Diabetes population}

A large national analysis investigating the comorbidity and its effect on 1590 patients with COVID-19 from 575 hospitals around mainland China showed that diabetes $(8.2 \%)$ is the second most prevalent comorbidity following hypertension (16.9\%), and its hazard ratio (HR) is 1.59 with $95 \%$ confidence interval (CI) between 1.03 and 2.45 after adjusting for age and smoking status [1]. An analysis of medical history,

Fuxue Deng and Dengfeng Gao contributed equally to this work.

Wei Jiang

xnkjiangwei@163.com

Shouping Gong

lecross@163.com

1 Department of Cardiology, The Second Affiliated Hospital of Xi' an Jiaotong University, Xi'an, Shaanxi, China

2 Department of Hematology, The Second Affiliated Hospital of Xi' an Jiaotong University, Xi'an, Shaanxi, China

3 Department of Infectious Diseases, The Second Affiliated Hospital of Xi'an Jiaotong University, Xi'an, Shaanxi, China

4 Department of Neurosurgery, The Second Affiliated Hospital of Xi'an Jiaotong University, Xi'an, Shaanxi, China symptoms and signs, demographic data, and laboratory and CT results from 174 consecutive patients with COVID-19 revealed that diabetes is a risk factor for COVID-19 and a poor prognostic predictor of the disease [2].

According to a 2010 large investigation report, the overall prevalence rates of diabetes and prediabetes are $11.6 \%$ and $50.1 \%$ in Chinese adults, respectively, which means that 113.9 and 493.4 million people have diabetes and prediabetes, respectively [3]. These values are currently believed to be much higher than those 10 years ago when COVID-19 outbreak in China at the end of 2019. Therefore, patients with diabetes and prediabetes who were infected with COVID-19 are a huge population in China and need close attention and care in treatment.

Diabetes attenuates the synthesis of inflammatory cytokines, such as IFN and interleukins. The downregulation of these cytokines impairs the balance of the host internal environment, shifts Th1 cells to Th2 cells, changes the normal function of macrophages and lymphocytes, and alters the function of endothelial cells of the mucosa in the front line of the innate immune system. All these changes weaken the barrier and immune system when a virus invades the human body. Dysregulation of the immune system may also account for the prolonged duration of the disease and delayed recovery in critical patients.

\section{ACE2 in diabetes}

As an important member of the renin-angiotensin-aldosterone system (RAAS), angiotensin-converting enzyme 2 (ACE2) plays a key role in the recognition and internalization of COVID-19 infection. ACE2 is widely expressed in multiple organs, including the pancreas, resulting in the development of insulin resistance or impaired insulin secretion [4]. In terms of ACE2 expression, diabetes is causally associated with upregulated ACE2 expression in the lungs, which may increase susceptibility to the new coronavirus. However, this hypothesis still needs to be confirmed in a future study. Insulin decreases ACE2 expression, whereas glucagon-like peptide-1 
(GLP-1) agonists and thiazolidinediones (TZDs) increase ACE2 expression [5]. Whether we should avoid TZDs in diabetes similar to the ACEI/ARB debate in hypertension also needs further research.

\section{The corticosteroid controversy}

Excessive systemic inflammation and multi-organ dysfunction are observed during pathogenesis caused by the coronavirus family, resulting in acute respiratory distress syndrome (ARDS) and septic shock. Inflammatory cytokine storms, such as IL-1 $\beta$, IL-6, IL-8, IL-10, and TNF- $\alpha$, also occur in COVID-19 infection during pathogenesis in critical patients. To reverse this situation, corticosteroids are widely used in clinical practice to decrease inflammation of the lungs and replace insufficient adrenal function when a physician encounters septic shock or ARDS.

In a large sample of 1099 patients with COVID-19 infection, $204(18.6 \%)$ received intravenous corticosteroids, and the percentage of corticosteroids was higher in severe patients than in mild or moderate patients [6]. For 52 patients with COVID-19 pneumonia in the ICU, 58\% were given intravenous glucocorticoids [7]. In 85 fatal cases, $76.5 \%$ patients received intravenous glucocorticoids, which was higher than that in common patients [8].

In clinical practice, low-dose (30-80 mg/day), short-term (3-5 days) methylprednisolone is a common treatment protocol, which was derived from the lesson of the severe acute respiratory syndrome (SARS) epidemic 17 years ago. However, a study showed that low-dose, short-term therapy does not exhibit significant benefits in inhibiting a cytokine storm and decreasing pulmonary exudation [9].

The efficacy of corticosteroids, especially in virus-related disease, has been disputed for a long time. An Expert Consensus on the Use of Corticosteroid in Patients with 2019-nCoV Pneumonia in China mentioned the dispute and controversy about corticosteroid usage for patients with COVID-19 and suggested that glucocorticoids should be administered with caution. Indications and dosage (methylprednisolone from $40 \mathrm{mg} /$ day to $160 \mathrm{mg}$ /day for 6 days according to the weight and status of the patients) should be strictly controlled in patients with COVID-19, and caution should be paid to patients with diabetes who receive insulin or oral anti-diabetic drugs [10].

To avoid the side effect of corticosteroid, new drugs, such as tocilizumab (a monoclonal antibody for rheumatoid arthritis), may be an alternative to control the cytokine storm instead of corticosteroids. The combination of continuous renal replacement therapy and tocilizumab may benefit severe patients in clinical therapy.

In conclusion, diabetes is a major comorbidity for COVID19 infection, and more attention should be paid to severe patients with diabetes who were infected with COVID-19. The administration of corticosteroids should be carefully determined for these patients, and suggestions from an endocrinologist should always be sought. Using other antiinflammatory drugs such as tocilizumab rather than potent corticosteroids should be an alternative. Once corticosteroids are administered, the side effects of corticosteroids may be harmful. Oral antidiabetic drugs should also be replaced by insulin therapy to counteract the side effects of hyperglycemia when corticosteroids are given intravenously. The blood glucose of hospitalized patients with diabetes should be monitored and adjusted in a timely manner to avoid a large fluctuation in blood glucose levels and deterioration of the disease.

Acknowledgments We acknowledge the Sino-French New City Branch of Tongji Hospital in Wuhan, PR China, and all patients with COVID-19 and healthcare workers who are fighting with this pandemic together.

\section{Compliance with ethical standards}

Conflict of interest The authors declare that they have no conflict of interest.

\section{References}

1. Guan WJ, Liang WH, Zhao Y, Liang HR, Chen ZS, Li YM et al (2020) China Medical Treatment Expert Group for Covid-19. Comorbidity and its impact on 1590 patients with Covid-19 in China: a nationwide analysis. Eur Respir J. https://doi.org/10. 1183/13993003.00547-2020

2. Guo W, Li M, Dong Y, Zhou H, Zhang Z, Tian C et al (2020) Diabetes is a risk factor for the progression and prognosis of COVID-19. Diabetes Metab Res Rev:e3319

3. Xu Y, Wang L, He J, Bi Y, Li M, Wang T, Wang L, Jiang Y, Dai M, Lu J, Xu M, Li Y, Hu N, Li J, Mi S, Chen CS, Li G, Mu Y, Zhao J, Kong L, Chen J, Lai S, Wang W, Zhao W, Ning G, 2010 China Noncommunicable Disease Surveillance Group (2013) 2010 China noncommunicable disease surveillance group. Prevalence and control of diabetes in Chinese adults. JAMA. 310:948-959

4. Bindom SM, Lazartigues E (2009) The sweeter side of ACE2: physiological evidence for a role in diabetes. Mol Cell Endocrinol 302:193-202

5. Muniyappa R, Gubbi S (2020) COVID-19 pandemic, corona viruses, and diabetes mellitus. Am J Physiol Endocrinol Metab 318: E736-E741

6. Guan WJ, Ni ZY, Hu Y, Liang WH, Ou CQ, He JX, Liu L, Shan H, Lei CL, Hui DSC, du B, Li LJ, Zeng G, Yuen KY, Chen RC, Tang CL, Wang T, Chen PY, Xiang J, Li SY, Wang JL, Liang ZJ, Peng YX, Wei L, Liu Y, Hu YH, Peng P, Wang JM, Liu JY, Chen Z, Li G, Zheng ZJ, Qiu SQ, Luo J, Ye CJ, Zhu SY, Zhong NS, China Medical Treatment Expert Group for Covid-19 (2020) China Medical Treatment Expert Group for Covid-19: clinical characteristics of coronavirus disease 2019 in China. N Engl J Med 382: $1708-1720$

7. Yang X, Yu Y, Xu J, Shu H, Xia J, Liu H et al (2020) Clinical course and outcomes of critically ill patients with SARS-CoV-2 pneumonia in Wuhan, China: a single-centered, retrospective, observational study. Lancet Respir Med:S2213-2600(20)30079-5 
8. Du Y, Tu L, Zhu P, Mu M, Wang R, Yang P et al (2020) Clinical features of 85 fatal cases of COVID-19 from Wuhan: a retrospective observational study. Am J Respir Crit Care Med 201:13721379. https://doi.org/10.1164/rccm.202003-0543OC

9. Liu K, Fang YY, Deng Y, Liu W, Wang MF, Ma JP et al (2020) Clinical characteristics of novel coronavirus cases in tertiary hospitals in Hubei Province. Chin Med J 133:1025-1031
10. Zhao JP, Hu Y, Du RH, Chen ZS, Jin Y, Zhou M et al (2020) Expert consensus on the use of corticosteroid in patients with 2019-nCoV pneumonia. Zhonghua Jie He He Hu Xi Za Zhi 43: $183-184$

Publisher's note Springer Nature remains neutral with regard to jurisdictional claims in published maps and institutional affiliations. 